\title{
The 3rd Asian Science Editors' Conference and Workshop 2016
}

\author{
Jieun Hani Kim \\ Tob Medical Translation, Seoul, Korea
}

Striving to advance scientific publishing in Asia, the Council of Asian Science Editors (CASE) organized the 3rd Asian Science Editors' Conference and Workshop 2016 in Seoul, Korea. This meeting report summarizes the proceedings of the annual 3-day event that I attended and informs readers of activities of editors in Asia.

\section{The Council and the Conference}

CASE conducted its 3rd annual event at the Korean Federation of Science and Technology Societies in Seoul between July 20 and 22, 2016. The event aimed at improving the quality of Asian scientific publishing was co-organized and -hosted by CASE and the Korea Institute of Science and Technology Information. Constituting of workshops and a conference, the event was a platform for editors from Asia to contemplate, discuss, and deliberate the issues of scientific publication, such as ethical publication practices and open access journals, relevant for Asian journals to leverage their efforts to push standards internationally. A total of 183 editors and administrators (mostly from the field of medicine) from 12 countries participated in the event. As participants from the hosting country, local editors composed the majority of participants (151 attendees). In descending order, participants flew out from Vietnam (7 attendees), Japan (4 attendees), and Philippines (3 attendees) to join in the conference.

Internationally renowned associations related to scientific writing, such as the American Medical Writers Association established in 1940 and the Council of Science Editors established in 1957, have a long history and have accumulated a wealth of knowledge. Compared to these long-standing associations, which together have a 134 years' history between them, CASE whose steps only began in 2014 has a meager 3 years behind it. The growth of scientific editors have occurred in step with that of the scientific publishing industry in the Western world, whereas in spite of an explosion of scientific research and publishing in Asia in the relatively recent decades the corresponding stride of scientific editors has been disappointing. Better late than never, the potential for growth for the Asian scientific publishing industry has become all the more anticipated and greater, and besides our start is so much more abundant, guided, and well-informed given the wealth of knowledge already built by our neighbors. Yet a much concerted effort is needed. To this end, organizing events where editors across Asia can assemble, discuss, and collaborate, which CASE has proactively spear-headed in Asia, is timely and ap- 


\section{science editing /}

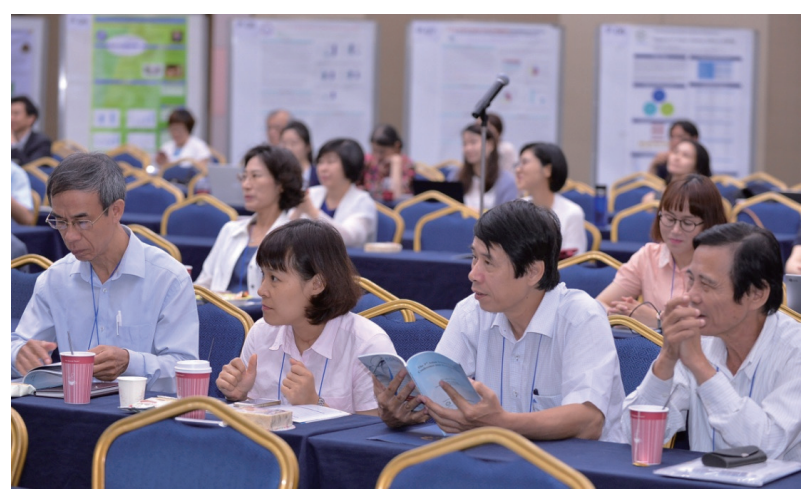

Fig. 1. Partakers of the conference who have come from various parts of Asia listen attentively to the afternoon sessions.

propriate. And the fast development of CASE is a confirmation of this (Fig. 1).

\section{Diverse Contents of the Event}

On Wednesday July 20, the conference kick-started with a workshop led by Rachael Lammey from Crossref, a company that assigns digital object identifiers (DOIs) to academic journals. The session gave the audience an understanding of Crossref's diverse services and provided an opportunity to hear first-handedly from Crossref their insights and their prospective services. An impending feature of Crossref's services was the pre-publication registration of DOIs; it was interesting to hear their stance on their decision to adopt a service that they had previously restricted. Furthermore, helpful and constructive tips pertinent to publishers were explained using specific examples. A back-to-back session from the Korean Journal of Medical Education allowed us to also see real experiences of using Crossref's services; many who listened to the talk were enlightened to see how these services when effectively and practically adopted correlate to measurable benefits, validating the effectiveness and the merits of Crossref services to many. All workshops of the first day were open to editors and editorial staff at no fee.

On the second day, the conference featured talks from a wide range of publishing-related organizations and those that discussed how open access and publication policies may be improved in Asia. The day began with an update on Crossref's current standing and future plans, followed by a presentation by Donald Samulack of Cactus Communications on the role of Asian editors to educate and inform authors of issues relating to unethical irresponsible publication practices and to predatory journals. As well as hearing broad and international perspectives of where the current status of scientific publishing lies in Asia, we were able to hear current perspec-

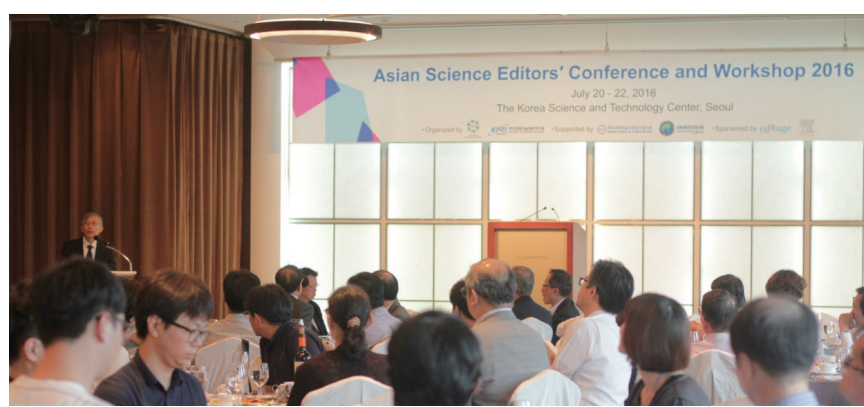

Fig. 2. Hyungsoon Kim welcomes guests to the evening reception.

tives at a country-wide scale, from Vietnam and Taiwan. Also, the importance of not neglecting local scientific journals was reminded, which is often overlooked in an ever globalizationseeking society. Moving back and forth between wide and narrow angles, the lectures covered a broad range of topics, no doubt keeping the audience alert and focused throughout. The day also dealt with various topical issues pertaining to open access. Current and up-to-date trends in Open Access were shared by Jungwook Seo, who had recently attended the 12th Berlin Open Access Conference. He described vividly the universal trend how journals are changing from subscription-based to open access systems.

On Friday July 22, the annual event wrapped up with workshops on the ISO (International Organization for Standardization) standard JATS (Journal Article Tag Suite) extensible mark-up language in online journal publishing. Taking Japanese and Korean publishing industries as examples, the sessions emphasized the importance of publishing in regional languages and addressed steps to ensure their survival in the publishing industry where the English language is universal. As on the first day, the last day's workshop was open to editors of all fields without need to register.

Apart from productive and informative sessions, the week gave us an opportunity to celebrate the gathering of editors and administrators through a reception that was held on the evening of the Conference. Celebratory messages were passed on by Donald Samulak and Hyungsoon Kim (Fig. 2). Guests were not only able to enjoy traditional Korean cuisine but also immerse in Korean culture by attending tourist and cultural visits prearranged by the organizers.

\section{A Promising Future for Asian Editors}

As possible harbingers of a flourishing industry, certain distinctive features of the Asian Science Editors' Conference and Workshop I felt set an auspicious tone for the future of Asian editors. First, attentiveness of the participants and the lively exchange of insights, comments, and advice between the 


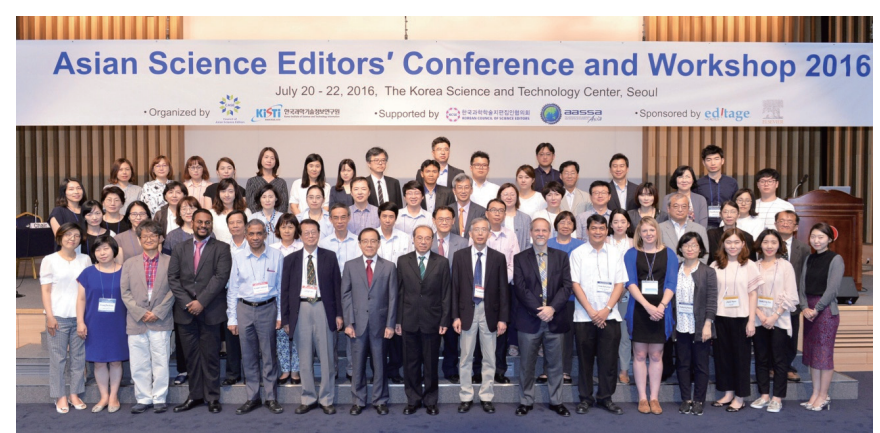

Fig. 3. Participants of the 3rd Asian Science Editors' Conference and Workshop 2016 gathered for a group photo.

speakers and the audience with whom they engaged was noticeable following every presentation. These 15 -minute-question and answer sessions provided sufficient time for speakers to communicate bi-directionally with the audience and vice versa. Second, the conference was attended by more multicultural participants than before (Fig. 3). For instance, although more than 400 participants from 15 countries participated in the 2015 annual event organized by the Council of Science Editors, the majority was from America and only $8 \%$ of par- ticipants were from abroad. In contrast, notwithstanding a smaller number of participants, almost a fifth of the participants of the 3rd Asian Science Editors' Conference and Workshop 2016 were from outside of Korea, not mentioning the invited speakers that had composed mostly of foreign speakers. Altogether the synergistic mix of these two factors-communication and diversity-makes me anticipate how future CASE conferences and similar events will shape scientific publishing in Asia.

The growth of CASE is a mark of the impetus that the field of Asian scientific publishing has received recently. Personally, the motley mix of speakers and participants from different nationalities and the lively discussions and exchanges amongst them during the event were an indication of this impetus. To conclude, I believe that moving forward in this momentum is an opportunity for the Asia's scientific publishing/editing industry to explosively grow and mature and to closely follow in the footsteps of their Western partners.

\section{Conflict of Interest}

No potential conflict of interest relevant to this article was reported. 